\title{
Nouvelles populations rurales et conflits au Québec : regards croisés avec la France et le Royaume-Uni
}

\author{
New rural populations in rural Quebec: \\ a comparative study with France \\ and the United Kingdom
}

\author{
Myriam Simard \\ Institut National de la Recherche Scientifique (INRS) \\ Urbanisation, Culture et Société \\ 385 Sherbrooke est, Montréal, Québec, Canada H2X 1E3
}

\begin{abstract}
Résumé
La recomposition sociodémographique des espaces ruraux s'est manifestée plus tardivement au Québec, comparativement en France et au Royaume-Uni. Après avoir tracé un bref bilan historique des écrits sur le sujet au Québec, l'auteure présente les résultats d'une recherche exploratoire qu'elle a réalisée en 2003 dans une région québécoise. Elle constate non seulement des tensions et conflits entre les diverses populations rurales (récentes et de longue date), mais également des alliances et solidarités autour d'enjeux communs. Ceci oblige donc à porter un regard plus nuancé sur les multiples interactions et dynamiques qui émergent dans la foulée de ces mutations dans les campagnes, et de scruter davantage les nouveaux espaces susceptibles de favoriser une mixité socioculturelle. Tout au long de l'article, l'auteure compare la situation québécoise avec celle de la France et du Royaume-Uni, y soulignant les convergences et divergences les plus marquantes.

(c) 2007 Lavoisier, Paris. Tous droits réservés.
\end{abstract}

\footnotetext{
*Adresse email : Myriam_Simard@UCS.INRS.Ca

doi:10.3166/ges.9.187-213 @ 2007 Lavoisier, Paris. Tous droits réservés.
} 


\section{Summary}

The sociodemographic redefinition of rural space is a recent phenomena in Québec compared to France and the United Kingdom. First, the author presents a brief historical overview of the related studies completed in Québec, followed by the results of an exploratory research which she undertook in a rural area of this province in 2003. Not only she notes the presence of tensions and conflicts amongst the diversified rural populations (recent and long-term residents), but she also observes cooperation and solidarity surrounding common issues. Considering the nature of these relationships, it is necessary to take into account the multiple interactions and dynamics which evolve with the transformations of the countryside. It is also essential to examine the new spaces likely to encourage sociocultural bond. In this article, the author compares the Québec situation with France and United Kingdom by underlining the most striking similarities and differences.

(C) 2007 Lavoisier, Paris. Tous droits réservés.

Mots clés : nouvelles populations rurales, recomposition sociodémographique des campagnes, conflits, alliances, nouvelle mixité socioculturelle, comparaison entre Québec, France et Royaume-Uni, migration ville-campagne, embourgeoisement rural.

Keywords: new rural populations, sociodemographic redefinition of countryside, conflicts, solidarity, new sociocultural bond, comparison between Quebec, France, United Kingdom, urban-rural migration, rural gentrification.

\section{Introduction}

On observe au Québec des transformations dans l'espace rural avec, notamment, l'arrivée de nouvelles populations tels des jeunes de retour après la fin de leurs études en ville, des retraités, des immigrants, des artistes, des travailleurs autonomes œuvrant à distance grâce aux nouvelles technologies de l'information'...Le contexte contemporain de mobilité accrue, la recherche de qualité de vie et de nouvelles modalités de réalisation professionnelle et personnelle expliquent, en partie, ces changements dans l'occupation du territoire rural. La persistance de cette recomposition sociodémographique des campagnes fait graduellement réaliser aux observateurs que cette migration de l'urbain vers le rural est un phénomène singulier ayant des incidences multiples sur la société rurale québécoise.

Alors qu'émergea en France et au Royaume-Uni, depuis près d'une vingtaine d'années, une production scientifique riche et abondante sur cette « renaissance » des campagnes pour reprendre l'expression de Bernard Kayser (1990), ces mutations commencent à peine à être analysées ici. Ce terrain de recherche est donc pratiquement vierge au Québec et demeure à développer, tel que je l'ai déjà signalé dans un article faisant le bilan des études rurales au Québec (Simard, 2002). Survenu plus tardivement au Québec qu'en Europe, ce mouvement de population vers l'espace rural demeure mal documenté et suscite plusieurs questionnements. Qui sont ces nouvelles populations? Pourquoi migrent-

\footnotetext{
${ }^{1}$ Par espace rural on entend ici les « régions rurales et petites villes (RRPV)» qui englobent les régions urbaines de moins de 10000 habitants et les régions rurales situées en dehors des zones de navettage des grands centres urbains (10 000 habitants ou plus) (du Plessis et al., 2002, p. 8). Le phénomène dont il est question dans cet article se distingue donc de la migration de citoyens vers les espaces périurbains des principaux centres urbains du Québec, tant par sa problématique que par son éloignement par rapport à ces centres urbains.
} 
elles vers l'espace rural ? Quel est leur processus d'insertion? Quelle est leur vision de l'avenir du monde rural? Leur présence suscite-t-elle des tensions et des conflits quant aux divers usages des espaces ruraux, comme cela fut démontré pour la France et le Royaume-Uni ? Y a-t-il des lieux de solidarité et de mixité entre les divers groupes de ruraux ? Comment se construisent alors de nouvelles formes de coopération entre des populations apparaissant à priori différentes au niveau de leurs trajectoires, de leurs représentations, de leurs attentes et de leurs intérêts ?

L'objectif de cet article est de présenter les premiers résultats d'une étude exploratoire sur le sujet réalisée en 2003 dans une région connaissant un repeuplement au sud du Québec, mais dans une zone suffisamment éloignée de l'espace périurbain de Montréal et de Sherbrooke. Mon but premier était de scruter ce nouveau phénomène, dans une perspective sociologique, pour en dégager les caractéristiques principales, notamment son impact global. Au centre de l'analyse se situent les diverses interactions entre les populations rurales, récentes ou de longue date, afin de donner une image plus nuancée et complète des liens sociaux dans le territoire et des processus à l'œuvre. Je voulais ainsi dégager l'ensemble des dynamiques sociales d'une société en profond changement, en n'écartant pas ses contradictions, ses hésitations, ses tensions et ses nouvelles alliances et solidarités afin de mieux saisir ses logiques et significations contrastées, un peu à l'image de l'analyse de Manceron en France (2005). Je désirais également mesurer à quel point l'espace rural, bien que mobilisé différemment selon divers groupes en présence, est capable paradoxalement de restructuration tout en maintenant une certaine cohésion sociale, certes renouvelée.

Cette démarche a permis de mettre en évidence non seulement des difficultés et des conflits, mais également des apports positifs et des collaborations contribuant à la revitalisation de l'espace rural. S'agit-il là d'une des spécificités du Québec rural où se discerne une oscillation entre conflits et collaborations, et même une complémentarité? Quelques pistes et éléments de réponses seront fournis dans cet article, en tentant de comparer les résultats lorsque possible avec ceux d'autres études en France et au Royaume-Uni où j'ai effectué récemment un stage sur cette thématique de recherche ${ }^{2}$. Ceci permettra d'apporter un nouvel éclairage, enrichi par les acquis de traditions scientifiques généralement isolées, et d'amorcer un pont entre la littérature française et anglo-saxonne sur le sujet. Dans une première section, sera donné un bref aperçu historique des écrits au Québec sur le sujet. Puis, dans une deuxième section, quelques repères méthodologiques de la recherche exploratoire seront rapidement présentés. Enfin, dans une dernière section, je me concentrerai sur les interactions variées entre les divers groupes des ruraux, en examinant tant les conflits que les collaborations et les nouvelles solidarités.

Avant d'aborder ces sections, il convient toutefois de faire une remarque préliminaire sur certaines caractéristiques du Québec rural, afin de favoriser une bonne compréhension de son contexte spécifique et d'éviter des comparaisons et des généralisations futures trop hâtives ou automatiques avec les espaces ruraux français ou britanniques. Ceux-ci

\footnotetext{
${ }^{2}$ Cet article fait suite à un ressourcement professionnel d'un an à l'étranger sur le thème de la recomposition sociodémographique des espaces ruraux. Survenu en 2004, il s'est déroulé principalement en France à l'Engref du Complexe Universitaire des Cézeaux de Clermont-Ferrand et, ensuite, en Écosse au Arkleton Institute for rural development research de l'Université d'Aberdeen. Que tous les membres de ces centres de recherche soient ici remerciés de leurs discussions stimulantes.
} 
présentent, en effet, d'importantes différences avec le Québec, spécialement au niveau des modes d'occupation et d'organisation des territoires, de la disponibilité des services, ainsi que des structures administratives et décisionnelles régionales et locales. Une faible densité de la population dans les régions rurales québécoises attire d'abord l'attention, couplée à l'ampleur de son territoire et aux grandes distances à parcourir habituellement entre les municipalités ${ }^{3}$. Rappelons qu'en 2001 le quart de la population québécoise, soit 1800000 personnes, demeure dans des collectivités à caractère rural et occupe $78 \%$ du territoire habité (Québec, 2005, p.8). En raison de la dispersion de la population et de la grande diversité du territoire où forêts, terres en culture ou en friche occupent la majeure partie de l'espace rural, les services sont inégalement répartis. Dans certaines localités affectées davantage par le dépérissement économique, l'exode des jeunes et le vieillissement de la population, quelques services de proximité de base sont menacés ou fermés. Enfin, notons qu'un processus de décentralisation est présentement en cours au Québec, avec diverses instances telles les 1100 municipalités rurales, les 103 municipalités régionales de comtés (MRC) et les récents conseils régionaux des élus (CRÉ) composés d'élus municipaux et de préfets des MRC. Les CRÉ sont les interlocuteurs privilégiés de l'État en matière de développement régional.

\section{Un phénomène récent au Québec et peu étudié}

Les travaux de deux pionniers ont principalement attiré mon attention par leur perspective originale axée sur le repeuplement rural. Parus tous les deux au début des années 1980, ce sont, à ma connaissance, les premiers écrits qui abordèrent directement le sujet (Brunet, 1980; McRay, 1981). Ils marquent ainsi une rupture avec le courant dominant de l'époque orienté vers les études sur la crise et le déclin des campagnes québécoises. Puisqu'une des préoccupations concernait l'avenir des terres agricoles, il n'est pas surprenant que les auteurs se soient attardés sur les incidences de l'installation de nouvelles populations sur le territoire agricole. Par le biais d'étude de cas, Brunet se concentre sur l'espace rural d'une région passablement éloignée de Montréal, à savoir les Cantons de l'Est, alors que McRay se consacre davantage à l'analyse d'un espace rural sous influence indirecte des deux grands centres urbains de Montréal et d'Ottawa. Les deux s'intéressent à la question de la poursuite d'activités agricoles par ces citadins migrant vers la campagne et qu'ils dénomment alors « ex-urbains » ou « ex-citadins ». Cette migration se situe dans le cadre du mouvement succédant à l'industrialisation et l'urbanisation de l'après-guerre et qu'ils qualifient de « repeuplement », de « retour à la terre », « d'exode urbain » ou « d'ex-urbanisation ».

Ces études sont intéressantes à double titre. D'une part, elles marquent l'émergence de nouvelles thématiques de recherche qui demeurent encore actuelles près de trente ans plus tard. Ainsi en est-il des motivations de migration en milieu rural et des diverses stratégies d'intégration. Est d'ores et déjà signalé le désir de ces nouveaux ruraux d'un nouveau «style de vie » (Brunet, 1980) ou « mode de vie » (McRae, 1981) où sont valorisés la vie rurale, les ressources de la nature, le silence, l'air pur...Ces notions s'apparen-

\footnotetext{
${ }^{3}$ Pour l'ensemble du Québec, comptant 7542760 habitants en 2004, on obtient une densité moyenne de 5,7 habitants au km². Cette densité varie d'un type de région à l'autre. Ainsi, elle affiche une moyenne de 0,9 pour les régions ressources situées en périphérie, de 33,0 pour les régions centrales parmi lesquelles se retrouve la zone étudiée lors de l'étude exploratoire, et enfin de 3 768,9 pour la région de Montréal (Québec, 2005, p.3).
} 
tent à la quête contemporaine d'une « qualité de vie », comme il sera vu plus loin. Elles apporteront un éclairage sur les divergences des divers acteurs ruraux- agriculteurs ou pas. D'autre part, ces deux études permettent de discerner la complexité des incidences de cet exode urbain, autant positives (conservation de l'environnement rural, création d'activités culturelles ou récréatives...) que négatives (hausse des taxes et du prix du foncier, ségrégation dans le réseau d'amitié, tensions...). Les rapports parfois conflictuels entre les agriculteurs et les ex-citadins sont soulignés : méfiance mutuelle, scepticisme, ambivalence des agriculteurs et critiques quant aux plaintes des ex-urbains au sujet des odeurs, du bruit, de l'emploi d'engrais chimiques, de la pollution de l'environnement... En outre, Brunet a le mérite de mettre en évidence l'hétérogénéité des ex-urbains en proposant une typologie ${ }^{4}$ qui apporte des nuances et des différenciations éclairantes pour la suite des études sur le sujet. Il y intègre, en effet, non seulement des individus adhérant à un changement de valeurs sociétales liées au phénomène culturel « anti-urbanisation », mais également une population marginalisée et à faible revenus qu'il dénomme « réfugiés » et qui « sont manifestement rejetés par des centres urbains où leurs ressources limitées ne leur permettent pas de s'établir de manière satisfaisante » (Brunet, 1980, p.399). Malheureusement, ce type de population précaire sera, ultérieurement, souvent oubliée et ignorée tant dans les études québécoises qu'européennes.

Les études subséquentes dans les années quatre-vingt poursuivront peu dans cette orientation des pionniers, étant plutôt préoccupées de dépeindre la déstructuration du monde rural et l'exode des jeunes au Québec. En témoigne la publication d'un numéro thématique de Recherches Sociographiques sur « le monde rural » en 1988 qui ne traite ni des nouveaux usages de l'espace rural ni d'un mouvement de repopulation rurale au Québec (Caldwell et Cohen, 1988). On note cependant une exception dans ces années avec Bernard Vachon (1986) qui, reprenant plus ou moins les mêmes notions des pionniers, observe que le renversement actuel de la tendance séculaire à l'urbanisation joue en faveur du repeuplement des régions rurales par des ex-urbains en quête d'un nouveau style de vie. L'accent n'est cependant pas mis sur les interactions entre ces nouveaux ruraux et les ruraux anciennement installés, ni sur les conflits ou collaborations qui peuvent en résulter. Plutôt, l'étude porte sur l'analyse statistique des recensements canadiens de 1971 à 1981 pour l'ensemble du Québec, afin de démontrer que la « désurbanisation » est loin de se limiter à un simple débordement métropolitain puisqu'il affecte aussi d'autres territoires ruraux même éloignés. Vachon s'attache alors à identifier ces nouvelles terres d'accueil et les divers facteurs explicatifs : évolution des valeurs et goût d'une vie naturelle, amélioration des réseaux routiers, flexibilité des horaires de travail, coût plus bas du foncier et des taxes hors des centres urbains, désir de passer la retraite dans un cadre rural... Cet auteur conclue qu'il s'agit bien plus «d'un retour à la campagne qu'un retour à la terre » et que «dans un avenir pas très lointain, la campagne apparaîtra peut-être aussi comme une terre d'accueil pour la nouvelle économie » basée sur les progrès de la technologie, le savoir et les services (Vachon, 1986, p. 92-93).

\footnotetext{
${ }^{4}$ Brunet retient cinq types d'ex-urbains : 1) les fermiers conventionnels; 2) les fermiers écologiques; 3) les fermiers écologiques potentiels; 4) les réfugiés; 5) les pseudos-banlieusards (Brunet, 1980, p.395-400). Quant à McRay, il distingue 2 types :1) les résidents non agricoles qui achètent de petites terres à des fins résidentielles; 2) les exploitants agricoles ludiques qui travaillent à temps partiel leurs terres (de plus de 12 hectares) (McRay, 1981, p.2).
} 
Vachon sera responsable au début des années quatre-vingt dix des travaux d'une équipe de chercheurs universitaires devant servir à préparer les État Généraux du monde rural qui auront lieu en février 1991. Bien que cet événement marque un tournant par rapport à l'attitude fataliste sur la désertification rurale et une profonde volonté du milieu local de se prendre en main pour esquisser un nouveau modèle de développement rural pour l'avenir, ces études demeurent encore timides et quasi silencieuses quant à l'arrivée, l'accueil et le processus d'intégration de nouvelles populations rurales au-delà des zones de périurbanisation. Les rares commentaires ne concernent, en effet, que les difficultés de cohabitation dans les espaces périurbains entre usages concurrents du territoire (agricole et résidentiel notamment) et entre population rurale de longue date et nouveaux résidents qui, eux, se sont installés dans la proche campagne à la faveur de la migration pendulaire (Vachon, 1991). Plus tard, l'idée est reprise de nouvelles opportunités offertes pour un renouveau rural, à la faveur de la tertiarisation de la structure économique et de la déconcentration émergente de ses activités, des nouvelles technologies, des télécommunications, du télétravail et des nouvelles valeurs sociales de qualité de vie, mais toujours sans aborder en profondeur la question des relations entre les divers groupes de ruraux quant à l'occupation de l'espace (Vachon, 1995; Vachon et Lemieux, 1997).

Ce n'est que plus récemment, à partir de la fin des années quatre-vingt dix ou au tournant du XXI ${ }^{\text {e }}$ siècle, qu'apparaissent des études centrées plus directement sur la transformation des populations rurales. Les démarches et perspectives adoptées sont diversifiées, les chercheurs provenant de plusieurs disciplines dont les principales sont l'aménagement du territoire, l'anthropologie, la géographie, la sociologie rurale, la sociologie du paysage...Sans prétendre en donner un portrait exhaustif ici, je me contenterai de souligner quelques grandes tendances émergentes. Les approches sont axées soit sur des études de cas à partir de villages ou de régions spécifiques (Domon et Paquette, 2001; Hébert et al., 2004; Pan et al., 1999; Roy et al., 2005; Simard et al., 2003; Solidarité rurale,2005a), soit sur des populations particulières en milieu rural- immigrants, artistes ou autres (Simard,1995; Solidarité rurale,2005b), soit sur une catégorie d'âge spécifique, à savoir les retraités (Groupe de réflexion d'UTA, 2001; Gagnon, 2005 ) ou les jeunes. Les travaux du Groupe de recherche sur la migration des jeunes québécois (GRMJQ) dont je suis membre ont, en effet, mis en lumière le « retour » de certains jeunes dans leur région d'origine après la fin des études en ville, et ce surtout pour des raisons de qualité de vie et d'attachement au territoire (Gauthier, 2003; Simard, 2003, 2004; Simard et al., 2001). Mais, encore là, aucune étude poussée n'est réalisée directement sur les interactions de ces jeunes dans le milieu d'origine, une fois le retour effectué. Le principal objectif du GRMJQ est, en effet, de scruter davantage les pratiques migratoires des jeunes et leurs motifs. Toutefois, des représentations négatives des jeunes sur l'inertie des décideurs et sur le contrôle de la région par les générations plus âgées laissent deviner certaines difficultés dans la cohabitation sur le même espace (Gauthier et al., 2006). Enfin, il convient de souligner les travaux constants depuis une dizaine d'années de l'équipe de Gérald Domon, associée à la Chaire en Paysage et Environnement de l'Université de Montréal, et qui se consacre à l'examen systématique des nouveaux rapports à l'espace rural et au paysage, notamment des relations entre les processus de migration rurale et le développement des 
paysages $^{5}$ (Paquette et Domon, 1999, 2003). Cette équipe s'efforce, entre autres, de dégager la diversité des situations spatiales, les nouvelles formes de ruralité et les dynamiques résidentielles qui en émergent. Tout comme Brunet, ces chercheurs relèvent la complexité des dynamiques de recomposition rurale, notant une polarisation entre deux profils de migrants urbains :1) ceux à la recherche d'un cadre de vie idyllique (rural idyll), généralement des professionnels âgés entre 45-64 ans ; 2) ceux, moins fortunés et moins mobiles, qui sont à la recherche d'un habitat rural à prix modique (Paquette et Domon, 2003, p.439).

L'ensemble de ces études récentes contribuent, par la diversité des thématiques abordées, à apporter des éléments nouveaux dans les connaissances. Ainsi en est-il pour les facteurs d'attraction et de rétention de ces nouvelles populations, leurs impacts multiplesautant positifs que négatifs- sur le territoire, leurs représentations de l'espace rural, leur rapport au paysage et les usages privilégiés de la campagne, les embûches et difficultés d'intégration, les conflits d'utilisation de l'espace rural avec la population locale et les autres acteurs. Il convient de noter qu'à cette même époque, des articles dans les médias traitent régulièrement de tensions entre agriculteurs et néo-ruraux (par ex : Lachapelle, 1999; Poissant, 1998). Cependant, aucune étude approfondie n'est réalisée pour donner un portrait d'ensemble de la diversité de ces nouveaux ruraux ni de leurs modes d'interactions avec les ruraux de longue date et autres acteurs-clefs locaux. Ceci aurait pourtant permis d'avoir une vision contemporaine de leur dynamique d'insertion et d'occupation du territoire et une meilleure compréhension des enjeux sous-jacents. Pareillement, aucune étude n'existe brossant un portrait de la situation dans les diverses régions du Québec, assurant d'avoir ainsi un portrait plus global. Beaucoup de questions demeurent donc en suspens et laissent dans l'ombre un large pan des phénomènes à expliquer quant à la diversification des populations et des usages en milieu rural et les réactions suscitées.

En résumé, les études québécoises sur le sujet n'en sont qu'à leur début et comportent beaucoup d'imprécisions qui affaiblissent leur portée. Les concepts sont ambigus notamment ceux désignant les nouvelles populations rurales, tels « ex-citadins », « néo-ruraux », «nouveaux arrivants ». La définition est parfois floue et associe à tort des sous-groupes qui pourtant devraient être distingués lors de l'analyse. Ainsi en est-il de la confusion chez certains entre villégiateurs saisonniers, touristes et nouveaux résidents permanents. Il devient alors difficile d'en tirer des conclusions plus générales. Il en est de même pour le concept de nouvelle ruralité qu'on associe parfois à d'autres concepts tout aussi vagues, tels société post-matérialiste ou post industrielle sans vraiment prendre la peine de bien les définir. S'impose ici la nécessité de clarifier le contenu de tous ces concepts pour en arriver à une meilleure compréhension globale et à une construction théorique et conceptuelle originale bien appropriée aux spécificités du Québec rural contemporain. Ces imprécisions associées aux diversités des approches rendent ainsi difficile la tâche d'en dégager une synthèse théorique et conceptuelle significative. La théorisation et la conceptualisation sur ce sujet y sont embryonnaires et les réflexions sont à poursuivre, comme le notent d'ailleurs Boyle et Halfacree (1998) pour la plupart des sociétés occidentales

\footnotetext{
5 À partir notamment d'une étude de l'évolution des trajectoires agricoles des municipalités rurales sur trente ans ( 1961-1991) et d'un examen des caractéristiques sociodémographiques actuelles des communautés rurales ( 1991), les auteurs dégagent trois grandes formes de ruralité sur le territoire étudié au sud du Québec : 1) le rural agricole; 2) le rural périurbain; 3) le rural de villégiature (Paquette et Domon, 1999, p.291-293).
} 
connaissant ce nouveau phénomène de repopulation rurale. Des ambiguïtés similaires ont d'ailleurs été à maintes reprises soulevées en France et au Royaume-Uni, révélant une fois de plus la complexité de la tâche, les risques de biais et d'exclusions et la difficulté de trouver une définition du rural issu de ces mutations (voir entres autres Boyle et Halfacree 1998; Champion et Watkins, 1991; Mathieu, 1990,1998; Perrier-Cornet, 2002a, 2002b; Philo, 1992; Schmitt et al., 2000; Thomsin, 2001).

\section{Une recherche exploratoire dans la région des Cantons de l'Est : quelques repères méthodologiques 6}

Cette idée de recherche sur les «néo-ruraux $»^{7}$ est née de mon observation personnelle depuis une vingtaine d'années de ce milieu de vie dans les Cantons de l'Est. J'y ai vu la ruralité se transformer au fur et à mesure que la population se diversifiait, de nouveaux besoins, services et usages du territoire apparaître graduellement, et plus récemment des nouveaux retraités arriver dans la région pour s'y établir définitivement. D'où le choix de concentrer cette première étude exploratoire sur le territoire de la municipalité régionale de comté (MRC) de Brome-Missisquoi situé au sud du Québec, aux limites des Cantons de l'Est et de la Montérégie, et partageant sa frontière avec le Vermont aux États-Unis. D'une superficie de $1537 \mathrm{~km}^{2}$, BromeMissisquoi comptait en 2001 une population de 46165 personnes réparties dans 20 municipalités ${ }^{8}$. À noter que la densité de la population au $\mathrm{km}^{2}$ y est de 30 personnes. Le territoire étudié accueille six villes régionales dont la plus populeuse est Cowansville avec 12032 habitants. Les autres municipalités y ont une population oscillant entre 250 et 2400 habitants. $^{9}$ On retrouve dans cet espace rural un patrimoine bâti d'influence loyaliste ${ }^{10}$ fort intéressant qui offre, avec les collines et les montagnes, un paysage des plus jolis et de plus en plus recherché comme cadre de vie. Déjà au XIX ${ }^{\mathrm{e}}$ siècle, cet espace était habité par une riche bourgeoisie anglophone

${ }^{6}$ Je remercie le Conseil de recherche en sciences humaines du Canada (CRSH-ARUC en Économie sociale) et le Ministère du Développement économique et régional du Québec pour leur support financier dans cette étude. Je remercie également mon équipe de recherche, Lucie Hébert et Anne Martin, pour leur collaboration dans ce projet en 2003, et Laurie Guimond pour sa participation à la mise à jour de la littérature en 2006.

${ }^{7}$ Contrairement à la France où la notion de «néo-rural » fut délaissée, cette terminologie fut utilisée pour cette étude au Québec puisqu'elle n'était pas autant connotée à la situation des étudiants parisiens contestataires de mai 68 qui, désirant s'affranchir du système de consommation, se sont établis en campagne (Léger et Hervieu, 1978).

${ }^{8}$ Source : Statistique Canada, 2002. Ottawa. Profils des communautés, Recensement de 2001. En outre, selon ces statistiques, on y dénombre un total de 22500 logements privés. À noter que près de 15000 habitants saisonniers s'ajoutent à la population totale (MRC Brome-Missisquoi. http://www.brome-missisquoi.ca/fr/mrc/ mission/presentation.html). La recherche a exclu ces résidents saisonniers puisque leur processus d'insertion relève d'une problématique différente.

${ }^{9}$ Source : Statistique Canada, Recensement 2001.

À titre d'indication, la distance entre Cowansville et Montréal est de 97 km, et de 95 km jusqu' à Sherbrooke. Celle de Sutton à Montréal est de 120 km, alors qu'elle est de 76 km jusqu'à Sherbrooke.

${ }^{10}$ Les loyalistes sont des individus ayant migré de la Nouvelle-Angleterre et de New York dans la région (alors Bas-Canada) entre 1775 et 1810 lors de la guerre d'indépendance américaine pour rester « loyaux » à la Couronne Britannique. Ils s'inspirèrent du style Nouvelle-Angleterre et de l'architecture anglo-saxonne de l'ère victorienne. 
qui y avait de luxueuses résidences, entre autres dans la municipalité du Lac-Brome (anciennement Knowlton) et à Cowansvillle"1( c.f. carte ).

Territoire de l'étude : MRC de Brome-Missisquoi (Québec)

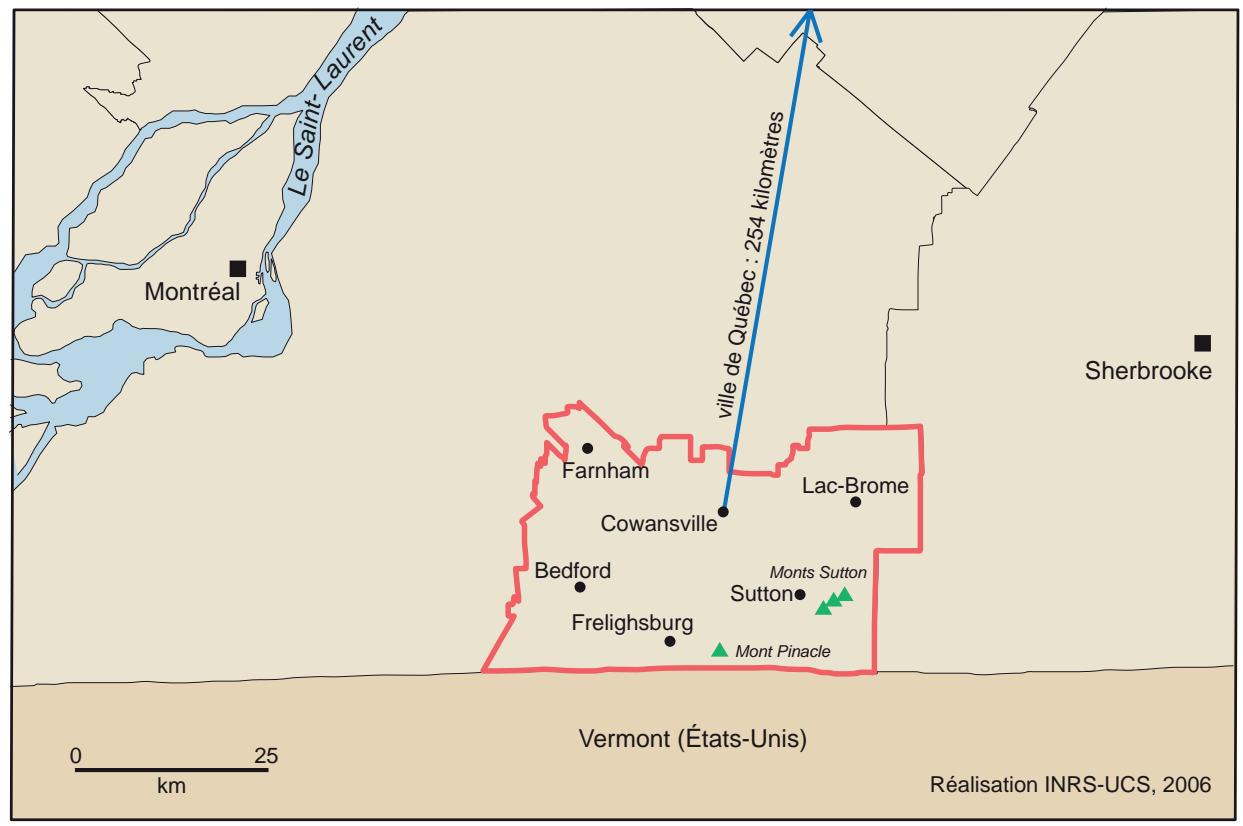

Situé en moyenne à environ une heure et demie de Montréal et de Sherbrooke, BromeMissisquoi est un territoire très différent entre ses secteurs Ouest et Est. À l'Ouest, se trouvent les plaines montérégiennes avec les municipalités de Farnham et de Bedford. Ces petites villes à vocation industrielle sont entourées de plusieurs petits villages agricoles où sont implantées des fermes importantes (maïs, élevage de porcs, de volailles, etc.). La population, à plus de $95 \%$ francophone, affiche un taux de pauvreté plus élevé que la moyenne québécoise et un niveau de scolarisation plus faible. Par contraste, le secteur Est avec le massif des Monts Sutton est plus montagneux. Le tourisme et la villégiature en sont les principaux moteurs économiques puisque le secteur manufacturier et le secteur agricole y sont beaucoup moins développés qu'à l'Ouest. La proportion de la population anglophone oscille entre $40 \%$ à $60 \%$ selon les villages. La scolarité ainsi que le revenu y sont plus élevés que la moyenne québécoise.

L'objectif de l'étude était d'analyser les diverses expériences autant culturelles, qu'économiques, sociales et environnementales implantées par et pour les «néo-ruraux » dans cette région et de dégager les différentes facettes de leur impact sur le milieu rural (Simard et al., 2003). Le rôle joué par les néo-ruraux dans ce territoire, leurs apports et difficul-

${ }^{11}$ Aujourd'hui, la population de Brome-Missisquoi compte $74 \%$ d'individus de langue maternelle française auxquels s'ajoutent $23 \%$ ayant l'anglais comme langue maternelle, alors que près de $3 \%$ ) ont une langue nonofficielle comme langue maternelle (Source : Statistique Canada, recensement de 2001). 
tés étaient au centre de l'examen, avec une attention particulière aux diverses modalités d'interactions entre les populations récentes et de longue date. À cet égard, plusieurs questions se posaient. Comment se vit l'interaction entre eux et les autres ruraux de plus longue implantation? Comment s'adaptent-ils mutuellement? La rencontre de ces divers types de villageois provoque-t-elle un choc de valeurs et des conflits? Y a-t-il un risque de clivage entre anciennes et nouvelles populations? Y a-t-il de nouvelles alliances et collaborations prometteuses?

Les diverses études européennes sur le sujet ont bien démontré la complexité et la difficulté de cerner et définir ces nouvelles populations rurales. Afin de mieux refléter la réalité spécifique et la dynamique de croissance du territoire étudié, une définition suffisamment large des «néo-ruraux » fut retenue. Celle-ci se réfère à une population qui a vécu en milieu urbain, incluant les migrants de retour et les ex-villégiateurs, et qui a fait le choix de vivre en permanence en milieu rural, pour des motifs d'ordre individuel, socio-économique ou parce qu'elle est fortement influencée par les qualités esthétiques et environnementales du milieu choisi. Partant de cette définition, près d'une vingtaine d'individus dits «néo-ruraux » furent rencontrés en 2003 en entrevue soit individuelle, soit de groupe (focus group). Ils provenaient de secteurs variés : secteur de développement économique (2); secteur environnemental (4); secteur culturel (5); autres secteurs (services de santé et sociaux, immigration, municipal, services aux jeunes) (5) auxquels se sont rajoutés de nouveaux arrivants retraités impliqués dans le milieu (3). Leurs témoignages ont permis de brosser un portrait plus diversifié que prévu.

En effet, on retrouve dans Brome-Missisquoi une population hétérogène aux parcours variés, incluant tant des jeunes ou des retraités de retour, des ex-villégiateurs établis définitivement en région rurale souvent à partir de leur retraite, des jeunes familles récemment installées, des immigrants, des artistes, des travailleurs autonomes et des nouveaux entrepreneurs profitant des nouveaux créneaux de services à offrir. La recherche d'une «qualité de vie » semble dominer comme motivation commune, avant les motifs d'ordre professionnel ou financier tout comme en Europe (Brun et al., 2002a; Cognard, 2001a ; Findlay et al., 1999 ; Guillot et Mamdy, 2004; Leader, 2000). Pour les interviewés, cette qualité de vie renvoie à plusieurs éléments tant objectifs que subjectifs, tels la convivialité, la beauté du paysage, le calme de la nature, la liberté et la sécurité, le soutien familial, l'accès à une maison, la possibilité de faire du bénévolat et d'avoir un emploi. Reste cependant le problème de savoir à partir de combien d'années de résidence permanente on cesse d'être un néo-rural, pour soi et pour l'autre ? Certains des néo-ruraux interrogés se considéraient, après un établissement de plus de 10 ans dans leur milieu, davantage comme des ruraux de longue implantation. Par contre, les études étrangères optent en général pour une durée d'une vingtaine d'années, les individus étant vus comme des résidents de longue date au-delà de cette période. Cette question demeure ouverte et mériterait d'être approfondie ultérieurement.

Au Québec, il semble que la plupart des milieux ruraux accueillent surtout des retraités comme nouvelles populations, bien que le profil commence à se diversifier davantage à l'image de ce qui est observé en France et au Royaume-Uni. Ainsi, les jeunes demeureraient encore minoritaires dans cette migration rurale, les opportunités d'emplois étant 
généralement insuffisantes pour les attirer davantage dans le Québec rural ${ }^{12}$. Rappelons que dans les diverses typologies élaborées dans ces pays européens nous observons l'inverse, alors qu'apparaissent prioritairement les jeunes familles avec enfants provenant surtout des classes moyennes urbaines, et que les retraités sont minoritaires. En raison de cette prégnance au Québec de retraités -et même de préretraités- issus majoritairement de la génération des «baby boomers » (ceux nés lors de la croissance démographique d'après-guerre), nous pouvons observer un certain «embourgeoisement » de l'espace rural, comme on le verra plus loin. Les chercheurs Britanniques furent d'ailleurs les premiers à évoquer ce phénomène avec leur concept de « rural gentrification » (Cloke et al., 1998 ; Fielding, 1998; Murdoch et al., 1998 ; Phillips, 1993, 2004 ; Smith et al., 2001). Curieusement, ils semblent n'avoir inspiré que peu d'auteurs Français qui l'appliquèrent alors parfois indirectement à la migration permanente de retraités d'Europe du nord dans les campagnes françaises, notamment des britanniques, hollandais et allemands (Barou et Prado,1995 ; Buller et al., 1994).

Cependant, tout comme certains chercheurs au Québec évoqués dans la section précédente, certains auteurs à l'étranger font également remarquer l'arrivée simultanée de populations dites « atypiques », plus vulnérables et marginales au niveau socio-économique (Cognard, 2001b; Garnier, 2001; Gilbert, 2002 ; Hugo et al., 1998). Cette situation, généralement occultée, fut également observée dans le territoire étudié avec la migration récente d'individus à statut socio-économique précaire. Ils proviennent de la métropole montréalaise ou d'autres centres urbains environnants et cherchent, notamment, des loyers à prix modique dans le secteur Ouest de Brome-Missisquoi. Ils ne représentent toutefois qu'une faible part des nouvelles populations rurales du territoire étudié.

\section{Des interactions diversifiées : entre conflits et collaborations}

Une pluralité d'interactions émergent de l'étude, traduites aussi bien par des conflits et tensions entre les diverses populations rurales que par des alliances et solidarités autour d'enjeux communs. Ceci oblige à porter un regard plus nuancé sur les multiples rapports sociaux susceptibles de se manifester suite à la diversification des usages et aux transformations sociodémographiques des campagnes québécoises. Ces usages sont, selon les distinctions apportées par Perrier-Cornet (2002a) de quatre ordres : productifs, résidentiels, récréatifs et de préservation. Cette « multifonctionnalité » induit inévitablement des rapports diversifiés, en encourageant des pratiques et des représentations sociales souvent antinomiques. Elle révèle une société à la fois dynamique et complexe où divers acteurs interagissent pour donner sens au territoire habité et inventer la société rurale de demain. Ces nouveaux usages de la campagne et les interactions variées qui y sont reliées nous forcent donc à poser un regard renouvelé sur le rapport ville/campagne, sur le rôle de chacun des acteurs ruraux et sur les modalités de faire «tenir ensemble » les diverses

\footnotetext{
${ }^{12}$ Afin de cerner à quel point le repeuplement rural concerne aussi les jeunes, je viens d'entreprendre une nouvelle étude dans une $2^{\mathrm{e}}$ MRC contrastée par rapport à Brome-Missisquoi, soit la MRC d'Arthabaska où les opportunités professionnelles sont plus nombreuses. Ceci permettra de dégager les convergences et divergences dans les processus d'insertion et d'interactions en milieu rural des jeunes, des adultes d'âges moyens et des retraités. Deux rapports présentent déjà les premiers résultats de recherche et sont disponibles sur le site internet de l'INRS (Guimond et Simard, 2007a, 2007b).
} 
composantes d'une société de plus en plus hétérogène. Les différents espaces de conflits et de collaborations exposés tour à tour ici permettront, je l'espère, d'apporter quelques éclaircissements sur les rapports diversifiés à l'espace rural et sur les modalités de restructuration de la société rurale québécoise contemporaine.

\subsection{Espaces de conflits}

Des conflits découlent inévitablement des fonctionnalités multiples de l'espace rural, à la faveur de la coexistence et de la concurrence croissante de plusieurs usages souvent incompatibles entre divers groupes : résidents permanents, occasionnels, agriculteurs, nouveaux ruraux... (Caron et Torre, 2002). Ils impliquent l'usage contradictoire de l'espace rural associé à la confrontation de conceptions divergentes à son égard. Quatre grands types de conflits apparaissent donc, liés à chacune des catégories d'usages (usages productifs, résidentiels, récréatifs et de protection) (Groupe Manon, 2005). Ces conflits se nouent souvent autour de la maîtrise du territoire, de l'accès inégalitaires à des ressources (eau, foncier agricole ou résidentiel...). Il y a alors résistance et contestation d'une appropriation exclusive de l'espace. Ces conflits révèlent des intérêts et des logiques antagonistes exacerbés par la complexification et la diversification des populations rurales d'aujourd'hui. Ils soulèvent la question de la légitimité de l'occupation effective ou proposée de l'espace. Ils éclairent en partie les mutations de l'espace rural et ses principaux enjeux. Ces conflits sont présents au Québec et traduisent selon le cas des divergences de représentations, des problèmes d'accès aux ressources ou des oppositions entre rationalités d'acteurs différents.

\subsubsection{Les visions divergentes autour de l'activité agricole et des projets de développement}

C'est surtout autour des usages productifs que surviennent la plupart des conflits en France et au Royaume-Uni (Caron et Rialland, 2001; Champion et Watkins, 1991; Daucé et Léon, 2002a; Nicourt et al., 2000). Le territoire étudié ne fait pas exception à cet égard puisque la production agricole et certaines de ses nuisances, principalement dans le secteur ouest, suscitent des réactions négatives de la part de certains nouveaux résidents venus chercher à la campagne la tranquillité et l'air pur. Les nuisances olfactives et sonores, la pollution des cours d'eau...sont notamment déplorées lors des entretiens, bien que ce sujet litigieux semble être évité par les plaignants dans la communauté pour éviter des tensions. Ainsi, l'agriculture intensive ne semble pas faire bon voisinage avec la vie saine souhaitée par ceux qui ont récemment choisi de résider dans un cadre de vie champêtre. Certes, ils acceptent aisément une agriculture bucolique et romantique avec quelques chèvres, poules ou vaches dans un paysage enchanteur, mais sans les contraintes liées au développement et à la revitalisation de la région. La représentation du monde rural de ces nouveaux ruraux ne concorde pas avec la réalité des pratiques agricoles actuelles, ni avec la nécessité de diversifier les formes de développement au-delà du tourisme et de la villégiature. Il y a plus de vingt ans, Claude Beauchamp faisait déjà remarquer une vision passéiste et idéalisée de la part des urbains au Québec ${ }^{13}$. Ceci est relevé en Europe où plusieurs auteurs ont mis en évidence la prégnance, parmi les nouvelles populations

${ }^{13}$ Beauchamp,C.,1982. Milieu rural et agriculture entre le rose et le noir. Recherches sociographiques 23 (3), 217-226. 
rurales, d'une représentation idyllique et stéréotypée de la ruralité qui vante les vertus de la vie villageoise souvent perçue comme homogène, conviviale, stable et quasi sans stress ni embûche. En France, on parle alors de la «folklorisation de la ruralité », alors qu'au Royaume-Uni on réfère à la « rural idyll » (Champion, 2001; Hervieu et Viard, 1996; Jedrej et Nuttall, 1996). Bernard Kayser (1990, p. 39) remarquait d'ailleurs chez le « néorural » un rapport à l'environnement différent de celui du paysan pour lequel «l'exploitation intensive du milieu reste un impératif de survie qui n'admet pas de contrainte extérieure ». Le principal défi des sociétés rurales est alors de trouver un équilibre entre ces visions opposées.

Un deuxième écueil relève de l'aménagement du territoire et de la planification de son développement, notamment au niveau touristique et récréatif. Ici aussi des conflits surviennent parmi les divers sous-groupes de ruraux. Bien que ces tensions ne se limitent pas à un simple clivage entre néo-ruraux et ruraux de longue date à ce sujet, il arrive que leurs intérêts respectifs soient assez divergents. Les uns voulant la tranquillité ou des projets minimalistes respectant le milieu naturel, les autres des activités plus complexes amenant la création d'emplois et la prospérité économique. L'exemple de la bataille du Mont Pinacle au début des années quatre-vingt dix en témoigne. Cette montagne, en l'occurrence la dernière à demeurer inexploitée dans la région, fut l'objet de débats virulents entre divers citoyens relativement à son développement en centre de ski privé. Avec sa cime rocheuse permettant une vue panoramique unique, elle représentait un patrimoine non seulement floristique et faunique avec des plantes médicinales au sommet et des nids de faucons pèlerins (espèce actuellement en péril au Canada), mais aussi un patrimoine historique et archéologique avec un cimetière d'amérindiens. Cette montagne, située dans la municipalité de Frelighsburg et jadis lieu de rencontre des indiens Abénaquis ${ }^{14}$, servait depuis quelques générations de lieu de randonnée pour les familles locales, surtout pour y admirer les couleurs automnales. Elle avait une valeur identitaire indéniable pour les citoyens et contribuait à consolider leur sentiment d'appartenance à une région unique.

Or, des promoteurs privés y achetèrent une majorité de terrains, entre 1989 et 1991, pour y faire un développement récréo-touristique de type club privé avec centre de ski alpin, condos de luxe, terrain de golf etc. D'un côté, plusieurs personnes du village y voyaient une opportunité de prospérité et de l'autre, plusieurs ruraux, dont une partie récemment installés, y voyaient une menace pour la qualité de vie et le patrimoine. En outre, ce développement représentait une menace pour les pomiculteurs environnants en raison des risques de pénurie d'eau occasionnés par les nouveaux services offerts par ce club privé (neige artificielle, arrosage des terrains de golf, usage domestique...). S'en suivit une longue bagarre entre ceux qui demandaient que le développement soit contrôlé et d'autre part, ceux qui voulaient qu'on laisse carte blanche aux promoteurs. La querelle se transporta même devant les tribunaux, ce qui mit fin au projet de développement, en raison notamment de frais juridiques élevés et de trop longs délais pour les promoteurs. Encore là, l'inquiétude quant à l'approvisionnement en eau et à l'impact sur l'environnement et sur l'ensemble de la communauté domina les débats. Certains nouveaux résidents

\footnotetext{
${ }^{14}$ Appartenant à la grande famille des Algonquins maritimes, ces amérindiens ont laissé notamment comme héritage bon nombre de noms de villages, de lacs et de rivières. Ainsi en est-il de Missisquoi qui signifie « de nombreux oiseaux aquatiques ». La région des Cantons de l'Est leur a servi « de zones de passage, de chasse, de trappage et parfois même de refuge » (Kesteman et al., p.60).
} 
et opposants au développement tentèrent même d'influencer l'issue de ce débat en investissant la politique municipale. Cette prise du pouvoir local par certains «étrangers » vint changer la vie politique locale, bouleverser les façons d'administrer habituelles et apporter des valeurs et règles nouvelles, à l'instar de ce qui fut observé en France et au Royaume-Uni dans des situations analogues.

Toute cette bataille provoqua de lourdes tensions parmi les villageois et résulta en un net clivage entre ceux favorables au développement annoncé et ses adversaires. Ceci laissa des séquelles dans la communauté pendant plusieurs années en fragilisant la cohésion sociale et en cristallisant les positions autour des rationalités opposées d'acteurs majeurs : promoteurs, associations de défense, élus municipaux... La complexité de la situation et des divers jeux d'alliances entre ces acteurs interdit cependant de réduire ces conflits à une simple opposition duale entre nouveaux ruraux et ruraux de longue date. Une telle conclusion serait précipitée et grossière et ne traduirait pas l'hétérogénéité des acteurs et des débats autour des enjeux cruciaux de l'aménagement du territoire et de l'orientation de son développement, particulièrement dans une zone à vocation touristique et de villégiature. Une réflexion reste à poursuivre à ce sujet puisqu'il s'agit là d'enjeux névralgiques au Québec comme le démontre le tollé d'oppositions survenues plus récemment, à la fin de 2004, au sujet de la construction d'un pylône de téléphonie cellulaire sur le même Mont Pinacle. Une mobilisation diversifiée ${ }^{15}$ parvint à faire arrêter le projet en évoquant notamment la nécessité de préservation de cette montagne et les incidences négatives sur les paysages. Ceci confirme à quel point le paysage semble être au cœur de conflits et de luttes aussi bien en France qu'au Québec (Gamache et al., 2004; Roussel, 2000). Cependant, cet enjeu paysager est paradoxalement porteur aussi de nouvelles alliances et solidarités entre citoyens, comme on le verra plus loin dans la section sur les espaces de collaborations.

\subsubsection{L'accès difficile à la propriété foncière en raison \\ de la hausse des coûts de l'immobilier}

L'arrivée de nouvelles populations rurales, notamment de retraités bénéficiant de bonnes rentes, a eu des conséquences négatives sur le prix de l'immobilier qui se mit à monter en flèche. Ceci a un impact désastreux sur l'installation des jeunes familles tant locales que venant de l'extérieur, puisqu'il leur est impossible d'acquérir des terrains ou maisons au prix du marché. Cet élément a été mentionné par presque toutes les personnes interrogées. Cette flambée des prix se remarque surtout dans la région montagneuse de l'Est de la MRC où les terrains et maisons sont devenus hors de prix, de sorte que l'accès à la propriété est maintenant pratiquement réservé aux nantis. En outre, ce phénomène entraîne inévitablement une pression sur la vocation agricole de la région. Le coût des fermes et des propriétés forestières devient trop élevé pour que ces dernières soient utilisées à des fins exclusivement agricoles ou forestières.

Ces problèmes d'accès à la propriété foncière s'apparentent à la situation de la France et du Royaume-Uni à cet égard. En particulier, plusieurs études furent réali-

\footnotetext{
${ }^{15}$ Entre autres, un blog fut créé sur Internet pour informer des enjeux et inviter les citoyens à protester par lettres et pétitions auprès des élus des divers niveaux, tant municipal que provincial et fédéral.
} 
sées sur les effets pervers quant au logement et à l'habitat en Angleterre ou au pays de Galles où la hausse des prix a, entre autres, contribué au départ des jeunes locaux incapables d'acheter (Cloke et al., 1991; Gallent et al., 2003; Shucksmith, 1991). En France, la sélection financière des habitants et l'exclusion des jeunes ménages sont déplorées (Mairie-Conseils, 2005). Au Québec, un sentiment de dépossession de la population de longue date se manifeste alors qu'elle voit ses jeunes partir à regret et ses terres achetées surtout par des individus fortunés arrivant de l'extérieur. Le concept d'embourgeoisement rural (rural gentrification), d'origine anglo-saxonne, s'avère ici fort pertinent. Se référant à un phénomène à la fois physique, économique, social et culturel, ce concept évoque une transformation des statuts d'occupation et une recomposition des propriétaires fonciers dans l'espace rural. Il implique le double processus de migration et de colonisation des classes moyenne et supérieure dans cet espace et le déplacement des classes plus modestes vers des lieux plus accessibles à l'extérieur. Cet embourgeoisement est lié à une demande croissante citadine des classes privilégiées de divers biens ruraux de consommation : construction/rénovation de maisons onéreuses, épiceries fines, produits de luxe variés...Ceci est de plus en plus visible dans le territoire étudié, notamment avec des produits alimentaires coûteux, la construction d'habitations spacieuses et cossues, des restaurants et auberges hautement cotés, un nombre croissant de voitures d'exportation haut de gamme devant les commerces usuels...

Ceci risque t-il de créer des inégalités et un clivage socio-économique et culturel dans la population, comme il fut observé dans d'autres pays (Cloke et Thrift, 1987; Clout, 1986; Raymond, 2004; Smith et Krannich, 2000)? Comment éviter un choc des valeurs et des conflits consécutifs? Peut-on parler d'un retour des classes sociales tel que récemment évoqué en France à la suite du renouveau des conflits sociaux et de l'extension des inégalités sociales (Bouffartigue et al., 2004) ? Ces questions méritent un approfondissement ultérieur à la lumière des pistes fournies par le concept d'embourgeoisement rural et les analyses sur les disparités sociales. À cet accès difficile à la propriété foncière, se couple dans BromeMissisquoi une absence drastique de logements locatifs. Ici aussi se remarquent des effets pervers au niveau de la rétention de jeunes et d'employés des diverses entreprises de services dans la région. Plusieurs emplois étant liés au tourisme et donc saisonniers, il est difficile de recruter du personnel pour de courtes durées en raison, notamment, de cette pénurie de logements. Cela vient accroittre encore plus le sentiment d'impuissance d'une partie de la population et son ressentiment par rapport à ces incidences néfastes d'exclusions.

\subsubsection{L'inadéquation entre les attentes des nouvelles populations rurales et les actions des élus}

Les nouveaux usages résidentiels et récréatifs de la campagne entraînent inévitablement des attentes inédites chez les nouvelles populations rurales. Or, la discordance entre les besoins exprimés par celles-ci et les actions des élus municipaux ressort de la recherche. Ainsi, quand on interroge des nouveaux résidents sur leur choix de lieu de vie, c'est d'abord la qualité de vie qui prime comme motivation principale avec la vie culturelle en tête de liste. En revanche, les projets culturels ne reçoivent pas toujours un bon accueil dans les conseils municipaux qui semblent, aux dires de certains interviewés, plus enclins à favoriser les activités économiques. En outre, est déplorée l'inertie des décideurs locaux à l'égard de la pénurie de services et d'activités pour les adolescents et les jeunes adultes 
de moins de 40 ans. L'accès à des ressources municipales diversifiées représente donc un des enjeux autour duquel se développent des insatisfactions et conflits.

Cette situation s'apparente à celle décrite en France où le rôle des élus locaux dans l'accueil et l'intégration des nouveaux arrivants est estimé insuffisant, notamment dans des sondages auprès des maires, des ruraux de longue date et des nouveaux résidents (Gramond, 2002,2003; Mairie-Conseils, 2005; Pineau, 2004; Sondage IPSOS, 2003). Une divergence entre les préoccupations de tous apparaît, en particulier sur les priorités à réaliser et les besoins des nouveaux. L'imprévoyance des élus et les déficiences dans les services municipaux sont reprochées. Le développement d'activités culturelles et de loisirs semble là aussi négligé par les maires. Dans l'Angleterre et l'Écosse rurales, des perceptions stéréotypées sur les nouveaux ruraux venant tant des autorités locales que de la population de longue date ont également été constatées, telle leur soi-disant tendance à s'emparer du pouvoir local, à s'objecter à tout futur développement, ou à ne pas chercher à s'intégrer (Henderson et Shucksmith, 1997; Stockdale et Findlay, 2004).

L'ignorance ou le manque de sensibilité des élus quant à cette problématique émergente au Québec, l'absence de politique d'accueil au niveau national et local, le peu de mention de cette nouvelle situation dans les diverses politiques ayant un impact sur le développement rural entraînent inévitablement une méconnaissance des besoins de l'ensemble des ruraux et une planification inadéquate et non conforme à la réalité $\mathrm{du}$ monde rural du début du XXI ${ }^{\mathrm{e}}$ siècle. Toutefois, la récente Politique nationale de la ruralité, adopté en décembre 2006 au Québec, accorde une plus grande importance à l'accueil et à l'intégration des nouvelles populations rurales ${ }^{16}$. La France, avant-gardiste à cet égard, a développé des politiques publiques inédites pour faciliter l'accueil et l'installation en milieu rural. Sa Charte nationale de l'installation en milieu rural, dont la ratification est imminente, mérite d'être signalée alors que rien d'équivalent ne semble exister au Royaume-Uni. Plusieurs écrits en France se concentrent, d'ailleurs, sur le processus d'accueil et d'intégration des nouvelles populations, entre autres sur les conditions de réussite telles la préparation et la réceptivité des territoires et la mobilisation de l'ensemble des acteurs locaux (parmi d'autres CVC, 2003, 2004; Exiga et Mamdy, 2004; Moquay et Roussel, 2002).

\subsubsection{Des conflits intergénérationnels autour du partage des coûts}

\section{d'équipements sportifs ou autres services}

Un autre conflit porte sur le partage des coûts des services régionaux et municipaux entre les différentes générations de citoyens. Un exemple récent, datant de 2003, concerne la querelle sur le partage des coûts de l'utilisation de la piscine de Cowansville entre jeunes résidents ayant des enfants à Sutton et une partie de ses retraités. Bien que le débat touche avant tout la question de la répartition des coûts d'infrastructures régionales entre les villes-centres et les villages utilisateurs environnants et déborde largement le simple conflit entre nouveaux et anciens ruraux, il indique des tensions entre certains groupes d'âges. Alors que les jeunes familles désirent que leur municipalité leur permette de bénéficier des équipements sportifs

\footnotetext{
${ }^{16}$ Ministère des Affaires municipales et des Régions, 2006. Politique nationale de la ruralité 2007-2014. Québec.
} 
et de loisirs diversifiés des villes voisines, des citoyens plus âgés y voient là une source inutile d'augmentation de leurs taxes municipales. Cette situation révèle une divergence quant à une vision commune de la ruralité de l'avenir où tant jeunes que vieux cohabiteraient. Ceci afin de créer un meilleur équilibre sociodémographique et une qualité de vie diversifiée pour à la fois attirer et retenir une panoplie de résidents ruraux ${ }^{17}$. Une expérience récente de parrainage réussi entre anciens entrepreneurs retraités et jeunes désireux de démarrer une entreprise dans la région vient, toutefois, atténuer cette opposition entre générations et laisse poindre des espaces de collaboration à cet égard.

Un aspect supplémentaire, mais plus indirect, concerne la pression sur les services sociaux et de santé de la région créée par l'arrivée de nouvelles populations et conséquemment sur ses coûts anticipés (CLSC, cliniques médicales, hôpitaux, agences sociales...). D'une part, la réalité de la hausse des prix n'est pas vécue avec la même intensité dans les villes et villages du territoire étudié. Ainsi, dans le secteur Ouest, la ville de Farnham connaît une migration des personnes les plus pauvres de la région montréalaise. En raison du coût peu élevé de loyers de Farnham et de la crise du logement des centres urbains à proximité, des citadins à faible revenu viennent alors s'établir dans cette ville. Ce phénomène crée ainsi une pression sur les divers services régionaux, aussi bien sociaux que de santé. D'autre part, l'arrivée des nouveaux retraités risque d'entraîner une plus grande demande de services de santé et d'hébergement de longue durée au fur et à mesure de leur vieillissement. Cette problématique fut déjà mentionnée dans la littérature étrangère qui fait ressortir, pour ces citoyens âgés, à la fois l'impact positif de leur engagement et de leurs expertises pour la revitalisation locale, mais également l'augmentation des coûts de santé et la nécessité d'une meilleure coordination entre les divers décideurs locaux pour organiser adéquatement ces services (Bryden, 2002; Callois et al., 2003). Ces nouveaux arrivants ne manquent donc pas de bouleverser inévitablement les modalités et les coûts de fonctionnement des organismes sociaux et de santé locaux qui doivent alors composer avec l'arrivée des nouvelles populations et les inclure dans leurs planifications futures. Sans créer des conflits immédiats et apparents, ce renouveau dans la population rurale accroît des pressions sur le système de santé et des services sociaux passablement déjà engorgé et affecté par une pénurie de médecins et de personnel spécialisé en régions au Québec ${ }^{18}$.

\subsection{Espaces de collaborations}

Les interactions entre les populations rurales, récentes ou de longue date, ne se limitent pas aux seuls conflits. Diverses alliances et coopérations s'observent sur le territoire étudié, donnant ainsi une vision plus globale des dynamiques sociales présentes. La société

\footnotetext{
${ }^{17}$ Se remarque ici une conception étroite d'un espace de vie « réservé » à certains groupes d'âge. Ainsi, un retraité nouvellement arrivé affirma que Sutton devait se destiner à l'accueil quasi exclusif de retraités et que les jeunes familles devraient quitter et déménager plutôt à proximité des villes où se trouvent les services et activités désirés.

${ }^{18}$ Sur cet aspect des difficultés de recrutement des médecins en régions, voir Myriam Simard et Nicolas Van Schendel, 2004, Les médecins immigrants et non immigrants en régions éloignées au Québec : processus d'insertion globale et facteurs de rétention, Cahier de recherche de l'INRS-UCS, Montréal, 230 p.
} 
rurale apparaît ainsi comme un espace ouvert et mouvant où la vie collective et les liens sociaux démontrent une capacité de renouvellement et d'ouverture aux changements. Ceci vient briser l'image stéréotypée de la société rurale québécoise présentée souvent comme traditionnelle et fermée sur elle-même. Ceci contribue également à la bonification du climat social et à la consolidation de la cohésion sociale.

\subsubsection{La préservation de l'environnement naturel}

et du patrimoine architectural et paysager

Les dernières décennies ont vu émerger au Québec, par l'entremise de divers groupes de pression, une claire volonté de préserver les sites naturels et le patrimoine architectural et paysager des campagnes, à l'instar de l'affirmation de l'usage de préservation portée notamment par des mouvements environnementaux en Europe. Ainsi, dans le territoire étudié, une série de fiducies foncières (inspirées des expériences de l'État voisin du Vermont aux États-Unis) et d'associations de conservation furent créées depuis la fin des années 1980 visant la protection de milieux naturels fragiles ou menacés : la fiducie foncière du marais Alderbrooke, le Parc d'environnement naturel de Sutton (PENS), la fiducie foncière de la vallée Ruiter, la fondation du marais Quillam-Durell, l'association de conservation du Mont Écho, la fiducie foncière du Mont Pinacle, le Corridor Appalachien, la fondation des Terres du Lac-Brome etc. Bien que ces groupes recrutent une bonne part de leurs membres parmi les nouveaux résidents, ils ne s'y limitent pas et constituent souvent un premier lieu de rencontres où une collaboration entre nouveaux et anciens ruraux peut se tisser. Ceux-ci partagent alors le même rapport à la nature, la même sensibilité à l'environnement et la même préoccupation de préserver les aspects qualitatifs et esthétiques de leur cadre de vie. L'appartenance et la mobilisation se fait ainsi bien plus sur la base des valeurs ou des intérêts communs que sur celle du milieu d'origine. Ceci paraît diverger avec la situation européenne où les diverses études font davantage ressortir les conflits et clivages entre populations nouvelles et de plus longue implantation à cet égard.

Un exemple récent de ces espaces de collaboration est la Société du temps libre dans Brome-Missisquoi, créée dans les années 90, et qui a dirigé récemment un séminaire sur le paysage en 2003. Ce séminaire a permis d'amorcer un mouvement de fond en s'associant au Parc d'environnement naturel de Sutton pour présenter une série de conférences sur le sujet. Ces deux organismes ont, ensuite, présenté ensemble le fruit de leur travail et de leurs discussions au conseil municipal de Sutton tout en travaillant en étroite collaboration avec le comité d'urbanisme. Ceci, afin de trouver des moyens de ralentir un développement accéléré qui menaçait la qualité du paysage. Des articles dans les journaux locaux ainsi qu'un dépliant de sensibilisation livré à toutes les adresses civiques de la municipalité vinrent compléter cette démarche. Il en résulta la création officielle au printemps 2004 du Groupe de réflexion et d'action sur le paysage et le patrimoine (GRAPP) dont la mission est de « préserver la richesse, la beauté et la diversité des paysages naturels et bâtis de la région au bénéfice des générations présentes et futures ». À moyen terme, il vise l'adoption d'une Charte de paysage régionale. Cet exemple illustre bien le potentiel de mobilisation de ces regroupements liés à l'environnement, où plusieurs acteurs locaux sont sollicités, aussi bien l'ensemble de la population que les élus municipaux et les 
dirigeants locaux. Il confirme que le rapport au paysage est complexe et peut à la fois diviser une population, mais aussi servir de catalyseur pour des acteurs partageant la même vision de sa préservation et mise en valeur.

Parallèlement, un mouvement de valorisation et d'accessibilité accrue aux sites naturels fut initié, au début des années 1990, pour améliorer l'accès de tous les citoyens aux beautés panoramiques du territoire. Bien que la communauté de Sutton habitait au pied des montagnes, il n'y avait paradoxalement que peu d'entrées publiques. La présence de plusieurs amateurs de plein air, le désir de développer des activités, tant pour les résidents locaux que pour les touristes et villégiateurs, ont incité le développement par divers organismes de 75 kilomètres de sentiers en boucle sur les Monts Sutton. En outre, une partie de la piste linéaire des Sentiers de l'Estrie traverse ce territoire et rejoint maintenant la longue piste renommée des Appalaches aux ÉtatsUnis (Appalachian Trail). Ceci contribue ainsi à ouvrir ce territoire à un vaste réseau nord-américain bien reconnu pour sa stratégie dynamique de conservation transfrontalière. Des sentiers ont également été aménagés sur le Mont Pinacle en remplacement du sentier traditionnellement utilisé depuis plus d'un demi-siècle par les familles locales, mais dont l'accès fut bloqué par des intérêts privés. Toutes ces initiatives donnèrent l'occasion à divers résidents ruraux - tant nouveaux qu'anciens - de travailler ensemble et de redessiner les liens sociaux. La composition de conseils d'administration de certains de ces organismes illustrent d'ailleurs bien ce travail en commun où siégèrent tant des personnes d'origine immigrée ayant une expertise en environnement et en gestion de sentiers pédestres acquise dans leur pays d'origine, que des résidents de longue date et des nouveaux ruraux venus de différents centres urbains du Québec.

\subsubsection{La création d'activités culturelles structurantes}

\section{pour le développement de la région}

Historiquement, la région des Cantons de l'Est a toujours manifesté une vie culturelle dynamique avec notamment une riche production musicale, littéraire et en arts visuels dès le XIX ${ }^{\mathrm{e}}$ siècle. Avec l'amélioration des moyens de transport et la popularité croissante de la villégiature, des artistes en arts visuels viennent séjourner dans la région, surtout pendant la saison estivale, et y constituent de petites colonies artistiques entre autres au Lac-Brome au début du $\mathrm{XX}^{\mathrm{e}}$ siècle. À partir de cette époque, plusieurs peintres de renom choisissent de s'installer dans la région, attirés nul doute par la beauté des paysages ${ }^{19}$. L'établissement soutenu d'artistes dans la région n'étonne donc guère puisqu'il est en continuité avec un mouvement amorcé depuis longtemps.

À la faveur de la migration de nouvelles populations vers la région étudiée, notamment de retraités et d'artistes, le besoin d'une vie culturelle vivante et diversifiée se fait sentir. Plusieurs nouveaux arrivants apportent alors leur contribution à la construction de nouvelles ressources culturelles. Différentes activités culturelles émergent tout en ayant des retombées non négligeables sur la vitalité tant économique, que culturelle et sociale de la région. Par exemple Arts Sutton qui existe depuis plus de 25 ans et qui administre une Galerie en arts actuels ouverte à l'année. Il compte ses membres non seulement dans la région mais

\footnotetext{
${ }^{19}$ Pour un aperçu de cette richesse culturelle, se référer à Kesteman et al. (1998). Voir également Gendron, M. (Ed), 1999. Histoire du Piémont des Appalaches, Presses de l'Université Laval et Éditions de l'IQRC, Québec.
} 
aussi plus largement à Montréal. Fondé d'abord par des artistes et soutenu tant par les membres artistes que les amateurs d'art surtout retraités, cet organisme a acquis une solide réputation. D'autres expériences sont également organisées, plus particulièrement liées aux arts visuels et où les artistes et artisans ouvrent leurs ateliers aux visiteurs à différentes saisons en les faisant sillonner les petites routes campagnardes (dénommées rangs au Québec) par un « circuit» des ateliers. Mentionnons le Tour des Arts en été pour la région de Sutton et du Lac-Brome; la Tournée des vingt pour Bedford-Frelighsburg ainsi que le Festiv'art à Frelighsburg l'automne. Également, les tableaux vivants organisés par Espace Sutton, des concerts dans des édifices patrimoniaux, de la musique et des contes dans les montagnes de Sutton, l'émergence de petites salles de spectacles telle Au coeur du village à Sutton... amènent un dynamisme culturel indéniable. Tous ces événements attirent beaucoup de touristes, valorisent les produits artisanaux et culturels locaux, sans compter qu'ils suscitent la rencontre entre diverses populations aussi bien rurales qu'urbaines.

Bien que les nouveaux ruraux semblent plus actifs dans ces divers événements culturels de la région, on observe, à l'instar des activités autour de la protection du patrimoine paysager et architectural, que leur mise en place offre l'opportunité à divers sous-groupes de villageois- anciens et plus récents- de se rencontrer, d'échanger des expertises, d'élargir des réseaux et d'amorcer des collaborations. Se dénote ici le désir de s'inscrire dans de nouvelles formes de développement par l'émergence d'entreprises culturelles en économie sociale. Ces projets, en plus de se situer dans une perspective de démocratisation, de solidarité et d'utilité sociale, concourent de façon originale à diversifier les pratiques du milieu, à consolider la cohésion sociale et à renforcer l'attractivité du territoire par son offre culturelle variée (Caillouette, 2002; CES, 2002; Lévesque et al., 1999). Ainsi, la réalité rurale et la moindre densité de la population incite les artistes et amateurs d'art à innover notamment par le réseautage et le partage d'expertises, de contacts et de ressources. Artistes et artisans en réseau coopératif (ARC) en est un exemple où des artistes professionnels ont créé une coopérative visant la mise en marché des œuvres des artistes locaux. Un représentant commercial fut embauché pour solliciter, au nom de tous les membres de la coopérative, le milieu corporatif afin de faire acheter leurs produits pour des événements spéciaux (Noël, retraite d'un employé, cadeau à un visiteur étranger... ${ }^{20}$.

Cette collaboration tend à se faire avec une certaine facilité compte tenu de la convivialité du milieu rural, bien que quelques entretiens aient révélé certaines tensions ou clivages au sein de la communauté locale. Mais, celles-ci semblent alors davantage dues à des divergences de visions au sujet du développement culturel rural (parfois entre francophones/anglophones, professionnels/non professionnels...) plutôt que le fait d'antagonismes entre nouvelles populations et ruraux de longue date. Cet aspect reste toutefois à approfondir dans l'avenir, en interrogeant autant les anciens ruraux que les nouveaux et en confrontant leurs expériences et perceptions. Pour terminer, il convient de souligner un dernier exemple de collaboration survenu dans la foulée d'une initiative culturelle et éducative de la Société du temps libre (STL). Développant une antenne de l'université du troisième âge à Sutton, rattachée à l'Université de Sherbrooke, celle-ci offre divers cours et conférences de qualité à la population locale. Malgré qu'elle soit fréquentée par une

\footnotetext{
${ }^{20}$ Je suis à finaliser une étude portant exclusivement sur le sujet et intitulée : Atouts, difficultés et défis des entreprises culturelles d'économie sociale en milieu rural : le cas de Brome-Missisquoi.
} 
majorité de nouveaux ruraux retraités, les cours particuliers sur l'histoire régionale du secteur ont connu un très bon succès également auprès des ruraux établis dans la région depuis longtemps. Cette double participation favorisa une occasion inédite de rencontres et de discussions entre les populations d'implantation ancienne et récente, et un point de départ pour des échanges ultérieurs sur les familles fondatrices des villages avoisinants, leurs parcours et apports diversifiés etc. Ceci montre bien qu'il existe des terrains de rencontres et de mixité sociale et culturelle présentement en émergence, mais que cela exige l'ouverture et l'imagination créatrice pour les concevoir et les mettre en place.

\subsubsection{La mise en place de services spécialisés jusqu'alors absents sur le territoire}

La participation, souvent bénévole, de plusieurs nouveaux résidents dans diverses activités communautaires locales figure comme un espace de collaboration, tel que constaté dans un séminaire organisé par la STL à Sutton (Groupe de réflexion de l'UTA, 2001). Ceci concerne une panoplie d'activités réalisées notamment par des retraités : bibliothèque locale, loisirs, visite des personnes malades, actions paroissiales, récupération d'objets usagés etc. Deux initiatives méritent d'être mentionnées, en raison du potentiel de collaboration qu'elles ont permis entre les diverses populations rurales, ancienne ou nouvelle. Il s'agissait, dans les deux cas, de mettre en place des ressources pour combler des besoins dans le territoire. La première concerne la création d'une résidence de transition pour la population âgée en perte d'autonomie qui devait, jusqu'alors, s'exiler pour aller en résidence pour ainés en dehors de la région. Pour éviter un déracinement de leur milieu de vie habituel, divers acteurs locaux s'appliquèrent à élaborer un projet de résidence à Sutton même, à partir de 2003 : conseillers municipaux, organismes locaux en santé ou en développement, bénévoles ayant des expertises diversifiées et pertinentes au projet etc. Réservée pour les 75 ans et plus, La Villa des Monts offre maintenant la possibilité de se loger dans la communauté. Dans un souci d'équité sociale, une partie des logements est réservée pour des personnes à revenu modeste. Une expérience identique, la Villa des Rivières, fut réalisée à Bedford.

La deuxième expérience concerne l'implantation d'une maison régionale de soins palliatifs pour malades en fin de vie et leurs familles, la Maison Au Diapason. Ici aussi, divers citoyens locaux sont impliqués sur une base bénévole : médecins, infirmières, gestionnaire, publiciste, architecte, décorateur, contracteur en construction...Cette initiative, présentement en cours dans la région, offre l'opportunité à plusieurs bénévoles aux expertises diversifiées de travailler ensemble pour l'intérêt général de la communauté. Une levée de fonds implique déjà la collaboration de plusieurs personnes, d'ancienne ou de nouvelle installation. Elle met en évidence la richesse et disponibilité locale en ressources humaines. Le nouvellement du capital humain en milieu rural ainsi que la valeur intégrative du bénévolat réalisé par des nouveaux arrivants dans la vie associative furent, d'ailleurs, soulignés en France et dans d'autres pays européens (Berger, 2004; Bryden et Hart, 2004; Findlay et al., 1998; Guérin et al., 2001).

\section{Conclusion}

Les exemples présentés dans cet article illustrent comment le rapport à l'espace rural peut à la fois réunir ou diviser sa population et contribuer, de façon originale, à la restructuration d'une société confrontée à de profondes transformations et à des représentations et logiques contrastées. En ce sens, non seulement les conflits mais aussi les nouvelles 
alliances sont des signes de ces changements qui traversent présentement la société rurale québécoise, notamment par rapport à l'occupation de l'espace et aux dynamiques sociales présentes. Ils traduisent les modalités contemporaines de partager un espace commun, avec tous les antagonismes, luttes, négociations, compromis et alliances que cela implique. Ils manifestent des mutations profondes dans les rapports sociaux et un renouvellement dans les modes de construction de la cohésion sociale.

Ces mutations peuvent être porteurs de renouveau à la condition de demeurer vigilant sur les dangers de clivages entre diverses populations rurales ayant des conceptions antinomiques de l'espace et des intérêts divergents. Ceci pose de nouveaux défis non seulement au milieu rural mais aussi à l'ensemble de la société et à l'État, autant au niveau démographique, qu'économique, culturel, social, environnemental et politique. Cela exigera des ajustements non seulement au niveau des ressources, tels les loisirs et l'accès au logement ou à la propriété, mais également au niveau des politiques d'accueil, d'intégration et de développement rural... Il s'agit, en fait, de l'important défi de la construction commune de la société rurale de l'avenir où une insertion de qualité sera possible. Dès lors, deviennent cruciales les stratégies émergentes de solidarité dépassant les conflits et aptes à créer une nouvelle mixité socioculturelle, entre divers acteurs, autour du développement du territoire rural commun.

Il subsiste plusieurs questions posées dans cet article. Une nouvelle recherche plus approfondie entreprise récemment sur le processus d'insertion globale (économique, sociale, culturelle et politique) des nouvelles populations rurales viendra apporter de nouvelles connaissances. ${ }^{21}$ Démarrée en 2006 dans deux régions contrastées au Québec, elle fait déjà ressortir la diversité des individus concernés, à l'instar de la situation en France et au Royaume-Uni (Guimond et Simard, 2007c). Cette recherche aura l'avantage d'inclure dans l'analyse, en plus des nouveaux ruraux, les résidents de longue date et les acteurs-clefs locaux (responsables d'associations villageoises, directeurs d'organismes locaux et régionaux, élus municipaux) absents dans la recherche exploratoire. Ceci permettra de mieux saisir la dynamique d'ensemble de la communauté rurale devenue de plus en plus complexe. L'étude permettra de voir si les défis de mobilisation de tous les acteurs et d'invention de modes inédits d'alliances et de rapprochements sont relevés. Par sa perspective non seulement diachronique (depuis l'arrivée jusqu'aux années subséquentes), mais aussi multigénérationnelle (jeunes, adultes, retraités), elle tentera de dégager les mécanismes aptes à atténuer les clivages et conflits et à favoriser la cohabitation harmonieuse des diverses populations rurales.

\footnotetext{
${ }^{21}$ Cette recherche est financée par le Conseil de recherches en sciences humaines du Canada $(C R S H)$ pour une période de 3 ans. Un volet comparatif est prévu avec, notamment, le reste du Canada et l'Europe.
} 


\section{Références}

Barou, J., Prado, P., 1995. Les Anglais dans nos campagnes. L'Harmattan, Paris.

Berger, A., 2001. Le rôle des mouvements migratoires dans les dynamiques rurales. In : Mamdy et al., (Eds), Fonctions d'accueil des territoires ruraux : les nouveaux arrivants. Actes du Séminaire du 22 mai 2001. Collection ACTES, n 6 .ENITA, 115-130.

Bouffartigue, P. (Ed), 2004. Le retour des classes sociales. Inégalités, dominations, conflits. La Dispute, Paris.

Boyle, P., Halfacree, K. (Eds), 1998. Migration into rural areas: theories and issues. John Wiley \& Sons Ltd, England.

Brun, A., Coulaud, D., 2002a. Le développement résidentiel dans deux communes de l'Orléanais. Dans : Perrier-Cornet, P. (Ed..), Repenser les campagnes. Éditions de l'Aube et Datar, Paris, 101-124.

Brunet, Y., 1980. L'exode urbain, essai de classification de la population exurbaine des Cantons de l'Est. Le géographe canadien XXIV (4), 386-405.

Bryden, J., 2002. The elderly outside the Metropolis: myths and realities. Conference: Haskell Master Class, Arizona State University, Arizona. http://www.asu.edu/xed/lectures/haskell02.pdf

Bryden, J., Hart K. (Eds), 2004. A new approach to rural development in Europe- Germany, Greece, Scotland and Sweden. The Edwin Mellen Press. Lampeter.

Buller, H., Hoggart, K., 1994. Vers une campagne européenne : les Britanniques en France rurale. L'Espace géographique 3, 263-273.

Caillouette, J., 2002. Des pratiques d'économie sociale en Estrie : le rapport à la dimension communautaire. Économie et Solidarités 33 (2), 41-53.

Caldwell, G., Cohen, Y. (Eds), 1988. Le monde rural. Recherches Sociographiques (numéro thématique) XXIX (2 \& 3).

Callois, J.-M., Mazuel, L., Roussel, V., Sencebe, Y., Vollet, D. et collaborateurs, 2003. Les retraités et les espaces ruraux du Massif central. Cemagref, Enesad, Enita, Clermont-Ferrand.

Caron, A., Torre, A., 2002. Les conflits d'usages dans les espaces ruraux : une analyse économique. Sciences de la société, no 57, 95-113.

Caron, A. et Rialland C., 2001. Recension des travaux portant sur les conflits d'usages dans l'espace rural et proposition d'éléments pour une typologie. Étude pour le Groupe de prospective espaces naturels et ruraux et société urbanisée. DATAR, France.

Champion, T., Watkins, C. (Eds), 1991. People in the countryside. Studies of social change in rural Britain. Paul Chapman Publishing Ltd, London.

Champion, T., 2001. The continuing urban-rural population movement in Britain: trends, patterns, significance. Espace, Populations, Sociétés 1-2, 37-51.

Chantier de l'économie sociale (CES), 2002. L'économie sociale et le secteur culturel. L'urgence d'agir pour soutenir la démocratie culturelle. Mémoire présenté à la ministre de la Culture et des Communications, Québec.

Cloke, P., Phillips, M., Rankin, D., 1991. Middle-class housing choice: channels of entry into Gower, South Wales. In: Champion T., Watkins, C. (Eds), People in the countryside. Studies of social change in rural Britain. Paul Chapman Publishing Ltd, London, 38-52.

Cloke, P., Philips, M., Thrift, N., 1998. Class, colonisation and lifestyle strategies in Gower. In: Boyle, P., Halfacree, K. (Eds), Migration into rural areas: theories and issues. John Wiley \& Sons Ltd, England, 166-185.

Cloke, P., Thrift, N., 1987. Intra-class Conflict in rural areas. Journal of Rural Studies 3 (4), 321-33.

Clout, H., 1986. L'évolution de la population rurale britannique. Espace, Populations, Sociétés 3, 19-32.

Cognard, F., 2001a. Reprise démographique et nouvelles populations dans les moyennes montagnes françaises. Espace, Populations, Sociétés, (1-2), 53-68.

Cognard, F., 2001b. Les nouveaux habitants des territoires ruraux. Dans : Mamdy, J.-F., Hausard, S. (Eds), Fonctions d'accueil des territoires ruraux : les nouveaux arrivants. Enita, Clermont-Ferrand, 35-45. 
Collectif Ville Campagne (CVP), 2004. Campagnes en mouvement. Synthèse de la rencontre des acteurs de l'installation en milieu rural du 15 et 16 décembre à Saint-Brieuc/Côtes d'Armor. Aixe-sur-Vienne.

Collectif Ville Campagne (CVC), 2003. Bilan des actions en faveur de l'accueil de nouveaux actifs en milieu rural, synthèse de l'étude réalisée pour la DATAR, Aixe-sur-Vienne.

Daucé, P., Léon, Y., 2002a. L'agriculture bretonne, un révélateur des tensions entre agriculture, environnement et cadre de vie. Dans : Perrier-Cornet, P. (Ed.), Repenser les campagnes, Éditions de l'Aube et Datar, Paris, 195-214.

Domon, G. et Paquette, S., 2001. Le paysage comme agent de recomposition des communautés rurale du sud du Québec : nouvelles possibilités, nouvelles exigences. Dans: Carrier, M., Côté, S. (Eds), Gouvernance et territoires ruraux : éléments d'un débat sur la responsabilité du développement. Presses de l'Université du Québec, Québec, 189-222.

du Plessis, V., Beshiri, R., Bollman, R. D., Clemenson, H., 2002. Définitions de «rural». Série de document de travail sur l'agriculture et le milieu rural. Document de travail $\mathrm{n}^{\circ}$ 61. Statistique Canada : Division de l'agriculture. Ottawa, p. 8.

Exiga, I., Mamdy, J.F., (Coord.), 2004. Dossier Cap sur la campagne, Pour 182, 51-243.

Fielding, T., 1998. Counterurbanisation and social class, In: Boyle, P., Halfacree, K. (Eds), Migration into rural areas: theories and issues. John Wiley \& Sons Ltd, England, 41-60.

Findlay, A., Short, D., Stockdale, A., 1999. Migration Impacts in rural England. The Countryside Agency, England.

Findlay, A., Stockdale, A., Short, D., 1998. The Impact of Migration in rural Scotland. Agricultural policy coordination and rural development research programme research findings no. 4. The Scottish office central research unit research findings, Edinburgh.

Gagnon, S., 2005. Les conflits d'utilisation du sol en espace rural : un problème fondamental de différenciation régionale à l'occupation « oisive » du territoire. Dans : Jean, B., Lafontaine, D. (Eds), Territoires et fonctions. Tome 2. Des pratiques aux paradigmes : Les systèmes régionaux et les dynamiques d'innovation en débats. CRDT et GRIDEQ, Rimouski, 205- 216.

Gallent, N., Shucksmith, M., Tewdwr-Jones, M. (Eds), 2003. Housing in the European Countryside. Rural pressure and policy in Western Europe. Routledge, London.

Gamache, N., Domon, G., Jean, Y., 2004. Pour une compréhension des espaces ruraux : représentations du paysage de territoires français et québécois. Cahier d'économie et de sociologie rurales, no 73, 72-102.

Garnier, E., 2001. L'installation des nouveaux résidents en espace rural: application au canton de Villeneuvede-berg en Ardèche. Dans : Mamdy, J.-F., Hausard, S. (Eds), Fonctions d'accueil des territoires ruraux : les nouveaux arrivants. Enita, Clermont-Ferrand, 69-84.

Gauthier, M. et al., 2006. La migration des jeunes au Québec. Rapport national d'un sondage 2004-2005 auprès des 20-34 ans du Québec, Cahier de recherche de l'Observatoire Jeunes et Société, INRS-UCS, Université du Québec, Québec.

Gauthier, M. (Ed), 2003. La migration des jeunes. Recherches sociographiques (numéro thématique) XLIV (1).

Gilbert, Y., 2002. La campagne recomposée : densification et recomplexification des sociétés locales en Languedoc-Roussillon. Revue d'Economie Méridionale, n 197-198, 123-136.

Gramond, F., 2003. Les citadins s'installent à la campagne. Synthèse du sondage sur les opinions et attitudes des néo-ruraux vis-à-vis de leur installation à la campagne en France. Ipsos, Canal Ipsos: les rendez-vous de l'actualité, Paris.

Gramond, F., 2002. Néo-ruraux: les citadins s'installent à la campagne. Synthèse du sondage sur les opinions et attitudes des maires de communes rurales en France vis à vis l'installation des citadins à la campagne. Ipsos, Canal Ipsos: les rendez-vous de l'actualité, Paris.

Groupe Manon, 2005. Horizons 2020: Conflits d'usage dans les territoires, quel nouveau rôle pour l'État? Rapport d'experts présenté au Commissariat général du Plan, France.

Groupe de réflexion de l'UTA, 2001. L'intégration des retraités urbains à la vie rurale. Texte rédigé dans le cadre du séminaire préparatoire au Congrès du $25^{\mathrm{e}}$ anniversaire de l'Université du Troisième Âge (UTA) de l'Université de Sherbrooke, Sutton. 
Guérin, M., Vollet, D. (Ed), 2001. Territoires ruraux et nouveaux venus. Collection Actes numéro 7. Éditions ENITA, Engref et Cemagref, Clermont-Ferrand.

Guillot, M., Mamdy, J.F. (Eds), 2004. Dossier/ Bilan Nouveaux acteurs et territoires d'accueil du Massif Central. Natamac, Clermont-Ferrand.

Guimond, L., Simard, M., 2007a. Nouvelles populations dans le Québec rural : le cas de la municipalité régionale de comté (MRC) de Brome-Missisquoi. Institut national de la recherche scientifique, Urbanisation, Culture et Société, Montréal. Disponible en ligne dans la rubrique «publication » sur le site de l'INRS-UCS à l'adresse suivante : http://www.inrs-ucsuquebec.ca/

Guimond, L., Simard, M., 2007b. Nouvelles populations dans le Québec rural : le cas de la municipalité régionale de comté (MRC) d'Arthabaska. Institut national de la recherche scientifique, Urbanisation, Culture et Société, Montréal. Disponible en ligne dans la rubrique «publication » sur le site de l'INRS-UCS à l'adresse suivante : http://www.inrs-ucsuquebec.ca/

Guimond, L., Simard, M., 2007c. Néo-ruralité au Québec : survol de la diversité des nouveaux ruraux dans Brome-Missisquoi. Journal of Eastern Townships Studies/revue d'études des Cantons de l'Est, n²9-30, (numéro double automne 2006 et printemps 2007).

Hébert, L., Simard, M., Martin, A., 2004. Les néo-ruraux. Un changement de paysage. Résultats d'une recherche exploratoire sur l'impact de l'arrivée de néo-ruraux à partir du cas de Brome-Missisquoi. CLD BromeMissisquoi, INRS et ARUC Économie Sociale.

Henderson, M., Shucksmith, M., 1997. In-Migration and rural communities. Rural Scotland today policy briefing. Rural Forum, Scotland.

Hervieu, B.,Viard, J., 1996. Au bonheur des campagnes. Éditions de l'Aube, La Tour d'Aigues .

Hugo, G., Bell, M., 1998. The hypothesis of welfare-led migration to rural areas: the Australian case. In : Boyle, P., Halfacree, K. (Eds), Migration into rural areas: theories and issues. John Wiley \& Sons Ltd, England, 107-133.

Jedrej, C., Nuttall, M., 1996. White Settlers. The impact of rural repopulation in Scotland. Harwood academic publisher, Luxembourg .

Kayser, B., 1990. La renaissance rurale. Sociologie des campagnes du monde occidental. Armand Colin, Paris.

Kesteman, J.P., Southam, P., Saint-Pierre, D. (Eds), 1998. Histoire des Cantons de l'Est. PUL et IQRC, Québec. (Coll. Les régions du Québec, 10).

Lachapelle, J., 18 février 1999. L'homme des chalets, Le Devoir, B1.

Leader magazine, 2000. A matter of quality of life. In: The (re) population of rural areas. European Observatory $22,13-15$.

Léger, D., Hervieu, B., 1978. Les immigrés de l'utopie. Autrement 14, 48-70.

Levesque, B., Mendell. M., 1999. L'économie sociale au Québec : éléments théoriques et empiriques pour le débat et la recherche. Lien social et politique - RIAC, No 41, 105-118.

Mairie-Conseils, 2005. Synthèse des résultats de l'enquête Mairie-Conseils 2004 sur « les nouveaux habitants des territoires ruraux ». Supplément à la lettre en Direct de Mairie-Conseils no 174, France.

Manceron, V., 2005. Une terre en partage. Liens et rivalités dans une société rurale. Éditions de la Maison des sciences de l'homme, Paris.

Mathieu, N., 1998. La notion de rural et les rapports ville/campagne en France : les années quatre-vingt-dix. Économie Rurale 247, 11-20.

Mathieu, N., 1990. La notion de rural et les rapports ville/campagne en France (1). Des années cinquante aux années quatre-vingts. Économie Rurale 197, 35-41.

McRae, J. D., 1981. L'établissement d'ex-citadins en milieu rural : Étude de cas dans la proche campagne de Montréal et Ottawa. Document de travail no 22. Environnement Canada, direction générale des terres, Ottawa.

Moquay, P., Roussel, V. (Eds), 2002. Les politiques d'accueil dans les territoires ruraux. Éditions ENITA, Collection Actes numéro 10, Engref et Cemagref, Clermont-Ferrand. 
Murdoch, J., Day, G., 1998. Middle class mobility, rural communities and the politics of exclusion. In: Boyle, P., Halfacree, K. (Eds), Migration into rural areas: theories and issues. John Wiley \& Sons Ltd, England, 186-199.

Nicourt, C., Girault J-M., Bourliaud J., 2000.Les odeurs d'élevages : textes, conflits et négociations locales. Economie Rurale, $\mathrm{n}^{\circ} 260,79-89$.

Pan, D., Domon, G., De Blois, S., Bouchard, A., 1999. Temporal (1958-1993) and spatial patterns of land use changes in Haut-Saint-Laurent (Quebec, Canada) and their relation to landscape physical attributes. Landscape Ecology 14, 35-52.

Paquette, S., Domon, G., 2003. Changing ruralities, changing landscapes: exploring social recomposition using a multi-scale approach. Journal of Rural Studies 19, 425-444.

Paquette, S., Domon, G., 1999. Agricultural trajectories (1961-1991), resulting agricultural profiles and current sociodemographic profiles of rural communities in southern Québec (Canada): A typological outline. Journal of Rural Studies 15, 279-295.

Perrier-Cornet, P. (Ed), 2002a. Repenser les campagnes, Éditions de l'Aube et Datar, France.

Perrier-Cornet, P. (Ed), 2002b. À qui appartient l'espace rural ? Éditions de l'Aube et Datar, France.

Phillips, M., 2004. Other Geographies of gentrification. Progress in Human Geography 28 (1), 5-30.

Phillips, M., 1993. Rural gentrification and the processes of class colonization. Journal of Rural Studies 2, 123-140.

Philo, C., 1992. Neglected Rural Geographics : a review. Journal of Rural Studies 8(2), 193-207

Pineau, J.-Y., 2004. L'accueil, un enjeu de société. Pour 182, 184-194.

Poissant, D., 26 juillet 1998. Le monde rural est en péril. La Presse, C4.

Québec (Gouvernement du Québec), 2005. Portrait socioéconomique des régions du Québec. Direction de l'analyse économique et des projets spéciaux, Québec.

Raymond, S., 2004. Des risques de marginalisation aux risques de gentrification: espaces ruraux convoités et inégalement accessibles. In: Arlaud, S., Jean, Y., Royoux, D. (Eds), Rural-Urbain. Nouveaux liens, nouvelles frontières. Presses de l'Université de Rennes, Rennes.

Roussel, V., 2000. À propos de l'arrivée de nouvelles populations et de ses conséquences sur les espaces ruraux. Revue d'Économie Régionale et Urbaine 1, 45-62.

Roy, L., Paquette, S., Domon, G., 2005. La campagne des néoruraux : motifs de migration, territoires valorisés et usages de l'espace domestique. Recherches sociographiques XLVI, 35-65.

Schmitt, B., Goffette-Nagot, F., 2000. Définir l'espace rural? De la difficulté d'une définition conceptuelle à la nécessité d'une délimitation statistique. Economie Rurale 257, 42-55.

Shucksmith, M., 1991. Still no homes for locals? Affordable housing and planning controls in rural areas. In: Champion T., Watkins, C. (Eds), People in the countryside. Studies of social change in rural Britain. Paul Chapman Publishing Ltd, London, 53-66.

Simard, M., 2004. Liens transnationaux et participation internationale des jeunes d'origine immigrée en région au Québec. Lien Social et Politiques 51, 111-122.

Simard, M., 2003. Le rapport à l'espace des jeunes issus de parents immigrés en région au Québec : un bricolage inédit? Recherches sociographiques (numéro spécial sur la migration des jeunes) XLIV (1), 57-91.

Simard, M., 2002. Espace rural et culture. Dans : Lemieux, D. (Ed.), Traité de la culture. Les Presses de l'Université Laval et les Éditions de l'IQRC, Sainte-Foy, 163-180.

Simard, M., 1995. Immigration agricole au Québec : impact sur le milieu rural et le développement régional. Revue canadienne des sciences régionales/Canadian Journal of Regional Science XVIII (3), 307-332.

Simard, M., Hébert, L., Martin, A., 2003. L’intégration des néo-ruraux dans Brome-Missisquoi : défis et recommandations. Actes de la $4^{\mathrm{e}}$ session de l'Université Rurale Québécoise (URQ), Mauricie, atelier sur les enjeux de l'arrivée des néo-ruraux et des immigrants dans le paysage, Québec.

Simard, M., Mimeault, I., Lévesque, M., 2001. Insertion en emploi et pratiques migratoires des jeunes d'origine immigrée en région au Québec. Dans : Roulleau-Berger, L., Gauthier, M. (Eds), Les jeunes et l'emploi dans les villes d'Europe et d'Amérique du Nord. Éditions de l'Aube, Lyon, 229-242. 
Smith, M.D., Krannich, R.S., 2000. Culture Clash Revisited : Newcomer and Longer Term Residents' Attitudes Toward Land Use, Development, and Environmental Issues in Rural Communities in the Rocky Moutain West. Rural Sociology, 396421.

Smith, D., Philips, D., 2001. Socio-cultural representations of gentrified Pennine rurality. Journal of Rural Studies 17, 457-69.

Solidarité rurale du Québec, 2005a. Étude de cas : Les Cerfs d'Alexis de Maskinongé. Recherche Migrations et développement rural. Québec. Document disponible sur le site web : www.solidarité-rurale.qc.ca

Solidarité rurale du Québec, 2005b. Deux études de cas: St-Adrien-de-Ham; Sainte-Clotilde-de-Beauce. Recherche Migrations et développement rural. Québec. Documents disponibles sur le site web: www. solidarité-rurale.qc.ca

Sondage Ipsos, 2003. Élus et néo-ruraux : divergences d'opinions sur l'installation. Village magazine 62, 20-26.

Stockdale, A., Findlay, A., 2004. Rural in-migration : a catalyst for economic regeneration. Conference Global Change and Human Mobility, Glascow.

Thomsin, L. (Ed), 2001. Repopulation et mobilités rurales. No thématique. Espace Populations Sociétés, 1-2.

Vachon, B., 1995. La mutation des espaces ruraux: nouvelles réalités, nouveau mode d'emploi. Dans : Côté, S. et al., Et les régions qui perdent...? GRIDEQ, GRIR, Département de géographie de l'UQAM, Québec, 357-377.

Vachon, B. (Ed), 1991. Le Québec rural dans tous ses états, textes produits aux États généraux du monde rural. Montréal, Boréal.

Vachon, B., 1986. Le peuplement des régions rurales du Québec face aux phénomènes de dénatalité et de désurbanisation. Espace, Populations, Sociétés 3, 85-94.

Vachon, B., Lemieux, A., 1997. Oser la renaissance rurale au Québec. Éléments pour une prospective des territoires ruraux. Hommes et Terres du Nord 3-4, 145-155. 
